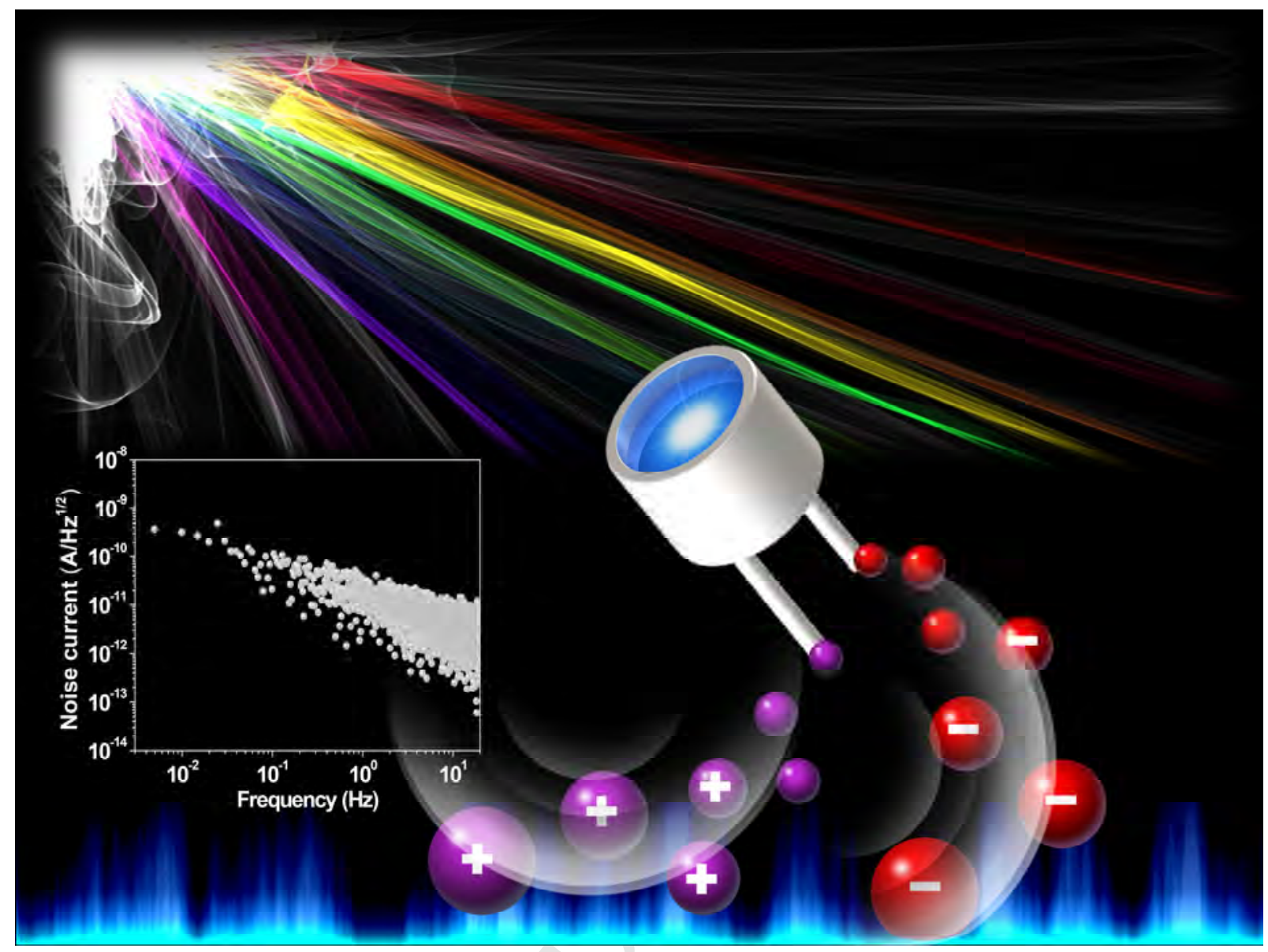




\title{
Detectivity Analysis for Organic Photodetectors
}

\author{
Il Ku Kim ${ }^{1}$, Jun Hyung $\mathrm{Jo}^{2}$, Brian (Jinwoo) Lee ${ }^{3}$, Young Jin Choi ${ }^{4}$
}

1. Centre for Integrated Smart Sensors (CISS), Korea Advanced Institute of Science and Technology (KAIST), Daejeon 34141, South Korea

2. School of Information and Communication Technology, Griffith University, South Port, QLD 4125 Australia

3. Civil Engineering and Built Environment School, Queensland University of Technology, Brisbane, QLD 4000 Australia

4. Department of Nanotechnology and Advanced Materials Engineering, Sejong University, Seoul, 143-743, Korea

\begin{abstract}
In this perspective letter, we report that there is a problem with a detectivity estimation method based on dark current measurement for organic photodiodes (OPDs). Based on dark current, calculated detectivity for fabricated OPD was $4.22 \times 10^{13} \mathrm{cmHz}^{1 / 2} / \mathrm{W}$ at $520 \mathrm{~nm}$. However, calculated detectivity for OPD based on measured noise current was $8.4 \times 10^{10} \mathrm{cmHz}^{1 / 2} / \mathrm{W}$ at $520 \mathrm{~nm}$. Therefore, we found that there are huge differences (more about 3 magnitudes of order) of detectivity calculations based on dark current measurement assumption and our noise measurement analysis. From the calculations of detectivities, it is concluded that noise current analysis should be addressed to clarify the organic photodiode characteristics.
\end{abstract}

Index Terms: Organic Photonics; Organic Compound; Photodiode; Luminescence Detector

\section{Introduction}

Organic polymer-based photodiodes (OPDs) are intensively studied for optical sensing and electro-optic applications [1]. They have intrinsic merits such as large-area photodetection, wide material selection opportunity and low-cost, low-temperature fabrication processing on flexible substrates [2]. One of the most important specifications of OPD is the detectivity, and it has been reported based on Gong's statement [3]. Gong et al., reported that 'If, as expected, the shot noise from the dark current is the major contribution, the detectivity can be expressed as

$$
D^{*}=R /\left(2 q J_{d}\right)^{1 / 2}=\left(J_{p h} / L_{\text {light }}\right) /\left(2 q J_{d}\right)^{1 / 2}
$$

where $q$ is the absolute value of electron charge $\left(1.6 \times 10^{-19}\right.$ Coulombs), $J_{d}$ is the dark current, $J_{p h}$ is the photocurrent and $L_{\text {light }}$ is the incident light intensity [3]. As Gong's statement, if shot noise is the dominant factor, it is the most desirable to reduce dark current for the enhancement of detectivity in the photodiode. However, the dark current-based assumption is not always true. From the report [3], the high-detectivity in PDDTT:PC ${ }_{60} \mathrm{BM}$ OPD was achieved by suppressing dark current using the blocking layer between the photoactive layer and the metal electrodes. However, unfortunately, their postulate and calculation of detectivity did not appropriately consider the critical electro-optical phenomena of photodiodes. When light is absorbed in the photo-active layer of OPD, an electron-hole pair (EHP) is generated in the photo-active layer. Also, this EHP generation is related to wavelength and optical power of the light. During EHP generation process, three types of noise are involved such as flicker (1/f) noise, Johnson noise and shot noise. Among three types of noise, shot noise is related to the statistical fluctuation in both high-optical power (photocurrent) and very low-optical power (dark current) of light. Another type of noise can be generated by thermal fluctuation which is called Johnson or thermal noise. Also, while measuring shot and Johnson noise, flicker (1/f) noise can be seen at low frequencies. Therefore, the origin of noise in the photodiode should be analyzed to accurately express the detectivity. Moreover, several recent reports on OPDs 
are still following the Gong's postulate [4-9]. The figure-of-merit of the photodiode is the noise equivalent power (NEP) to distinguish between detected minimum optical power and the noise. However, recently published literatures have not fully considered the advantages of the NEP properties for the detectivity calculation. To address this critical issue, we have calculated this detectivity for both OPD made from the conventional device architecture and a Si-based photodiode. We measured dark current density $\left(J_{d}\right)$, noise current $\left(i_{n}\right)$ and external quantum efficiency (EQE), then calculated detectivities and NEPs based on both $J_{d}$ and $i_{n}$. In addition, a generalized transfer matrix method (GTMM) calculation results will be introduced to define the behavior of light absorption in the OPD structure [10-11].

\section{Experimental Details}

In this study, OPD device was fabricated with a donor-acceptor blend of poly(3-hexylthiophene-2,5-diyl) (P3HT) and [6,6]-phenyl-C60-butyric-acid-methyl-ester $\left(\mathrm{PC}_{60} \mathrm{BM}\right)$ for a planar photo-active layered structure. P3HT and $\mathrm{PC}_{60} \mathrm{BM}$ were dissolved at $70{ }^{\circ} \mathrm{C}$ in 1,2-dichlorobenzene (DCB) and stirred for 12 hours. A purchased (Kintec Company) indium tin oxide (ITO) coated glass substrate was cleaned sequentially by ultrasonic treatment in detergent, de-ionized water, acetone and isopropyl alcohol. A thin layer of PEDOT:PSS (20 nm) was deposited on top of the ITO-coated glass substrate by a spin-coating method with speed of 4,000 rpm for $40 \mathrm{sec}$. Then the P3HT: $\mathrm{PC}_{60} \mathrm{BM}$ bulk-heterojunction $(\mathrm{BHJ})$ layer was spin-cast from the blended solution to form a thickness of $120 \mathrm{~nm}$. The total concentration of P3HT: $\mathrm{PC}_{60} \mathrm{BM}$ blend was $30 \mathrm{mg} / \mathrm{ml}$. Finally, a $\mathrm{C}_{60}(40 \mathrm{~nm})$ hole-blocking layer $(\mathrm{HBL})$ and $\mathrm{Al}(100 \mathrm{~nm})$ electrode were deposited on top of the BHJ layer. The OPD surface area was $0.2 \mathrm{~cm}^{2}$. Figure 1 shows fabricated OPD structure and band diagram for detectivity evaluation. In the case of $\mathrm{C}_{60}$ layer, it has applied to reduce the dark current density level as reported [1]. All devices were annealed at $150{ }^{\circ} \mathrm{C}$ for $10 \mathrm{~min}$ in $\mathrm{N}_{2}$ filled glove box. All OPD devices were electrically characterized in the air after encapsulation. A commercial characterization system for $J-V$ and EQE was supplied by PV Measurement Inc. to acquire the data. The noise currents were measured with Agilent B1500A as reported by Kim et al. [12]. Si-PD (D214) was purchased from Hamamatsu. A GTMM simulation was accomplished to calculate the absorption fraction and charge generation rate in the multi-layered interface of OPD. To perform GTMM analysis, optical constants such as reflective index $(n)$ and extinction coefficient $(k)$ values were obtained after Ellipsometry measurements.

(a)

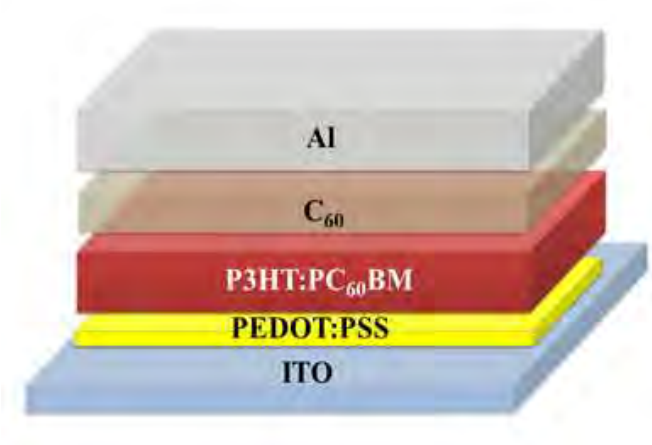

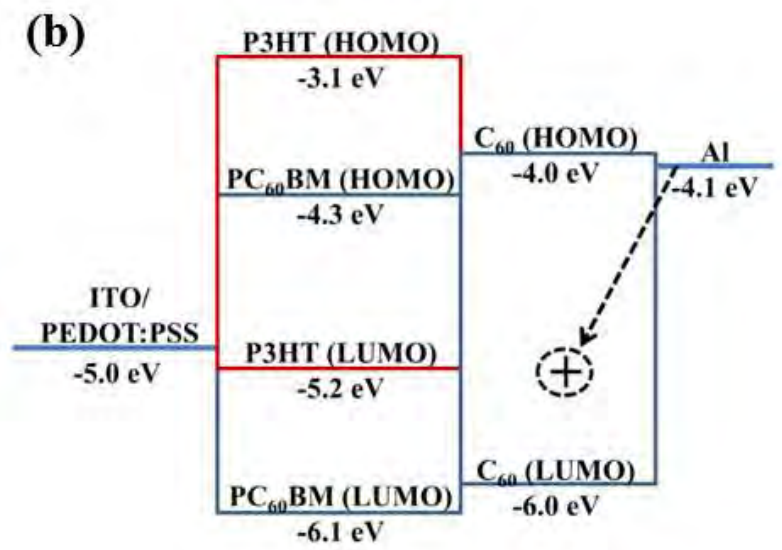

Fig. 1 a) Fabricated OPD structure, b) band diagram for fabricated OPD.

\section{Results and discussion}


Figure 2 shows measured $J-V$ results under the dark condition for both Si-PD and OPD. For Si-PD, measured dark current densities were $6.0 \times 10^{-8} \mathrm{~A} / \mathrm{cm}^{2}$ at $-1.0 \mathrm{~V}$ and $3.5 \times 10^{-11} \mathrm{~A} / \mathrm{cm}^{2}$ at $0 \mathrm{~V}$ conditions, respectively. In the case of OPD, measured dark current densities were $1.6 \times 10^{-8} \mathrm{~A} / \mathrm{cm}^{2}$ at $-1.0 \mathrm{~V}$ and $8.1 \times 10^{-11} \mathrm{~A} / \mathrm{cm}^{2}$ at $0 \mathrm{~V}$ conditions, respectively. Note that, dark current level of OPD in reverse bias condition is better than commercialized Si-PD and $\mathrm{C}_{60} \mathrm{HBL}$ is effectively working.

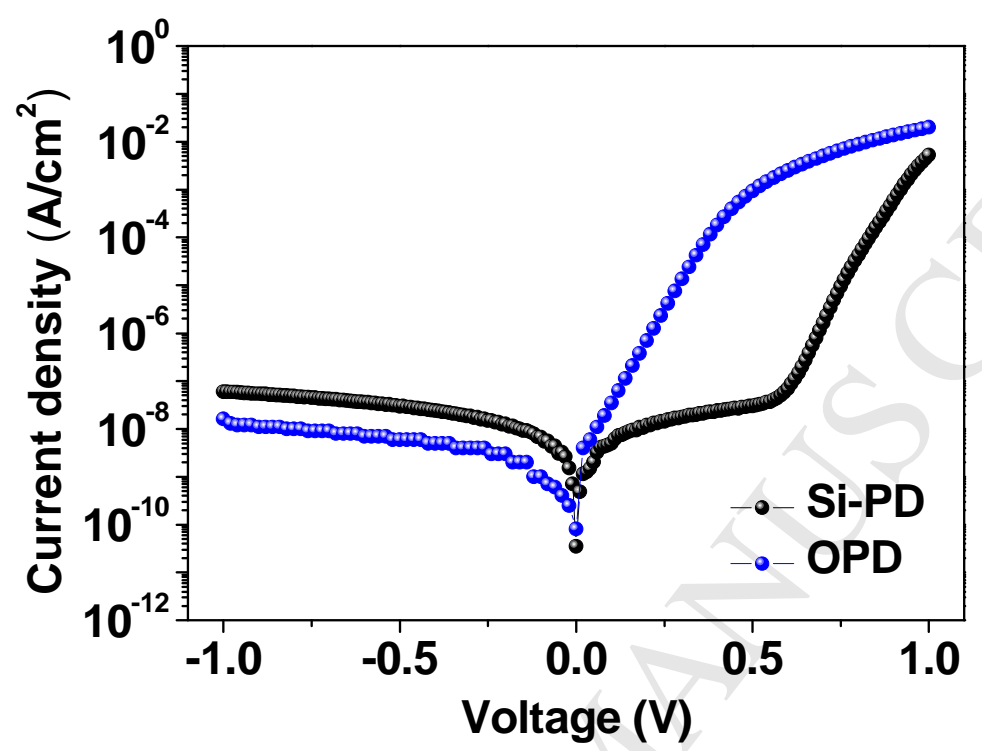

Fig. 2 Measured $J-V$ results under the dark condition for Si-PD and OPD.

Figure 3 confirms charge generation and extraction performance by GTMM calculation. In the bulk-heterojunction (BHJ) of $\mathrm{P} 3 \mathrm{HT}: \mathrm{PC}_{60} \mathrm{BM}$ blend, maximum charge generation is occurred near the interface of PEDOT:PSS and $\mathrm{C}_{60}$ layers, respectively. Then generated charges are extracted by built-in voltage potential for both anode and cathode electrodes before recombined [1].

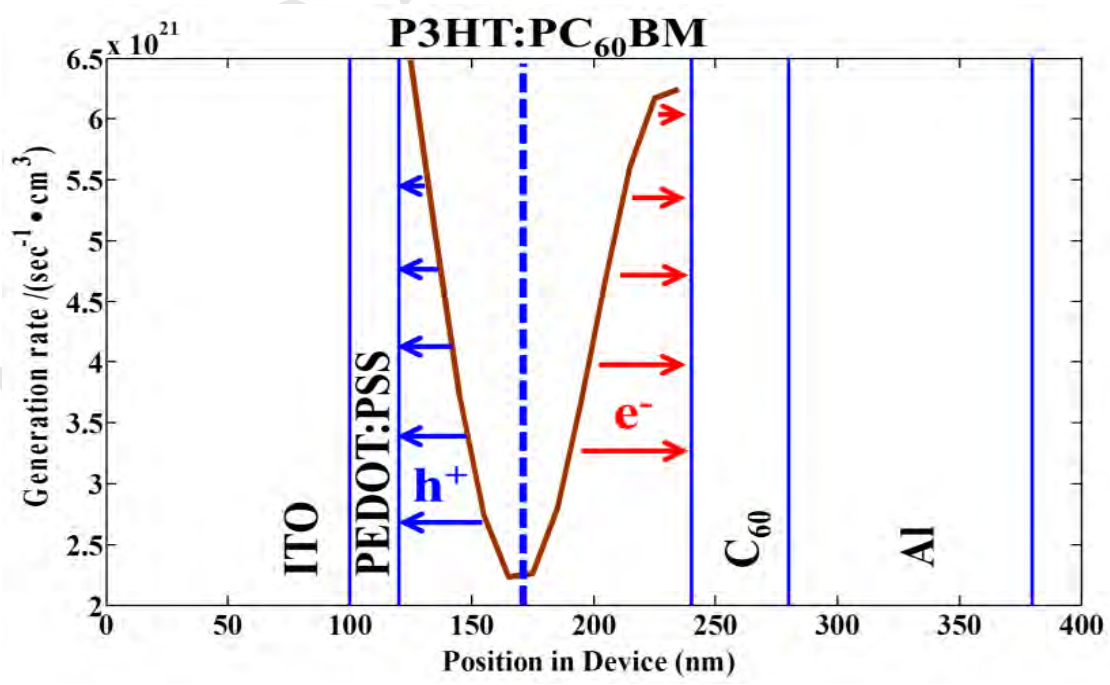

Fig. 3 Charge generation simulation result by generalized transfer matrix method (GTMM).

Figure 4 shows measured EQE results for both Si-PD and OPD at zero bias condition. As expected, the photo-response of $\mathrm{Si}-\mathrm{PD}$ covers the spectral range up to 1,100 $\mathrm{nm}$. However, OPD has frail photo 
response after $700 \mathrm{~nm}$ wavelength.

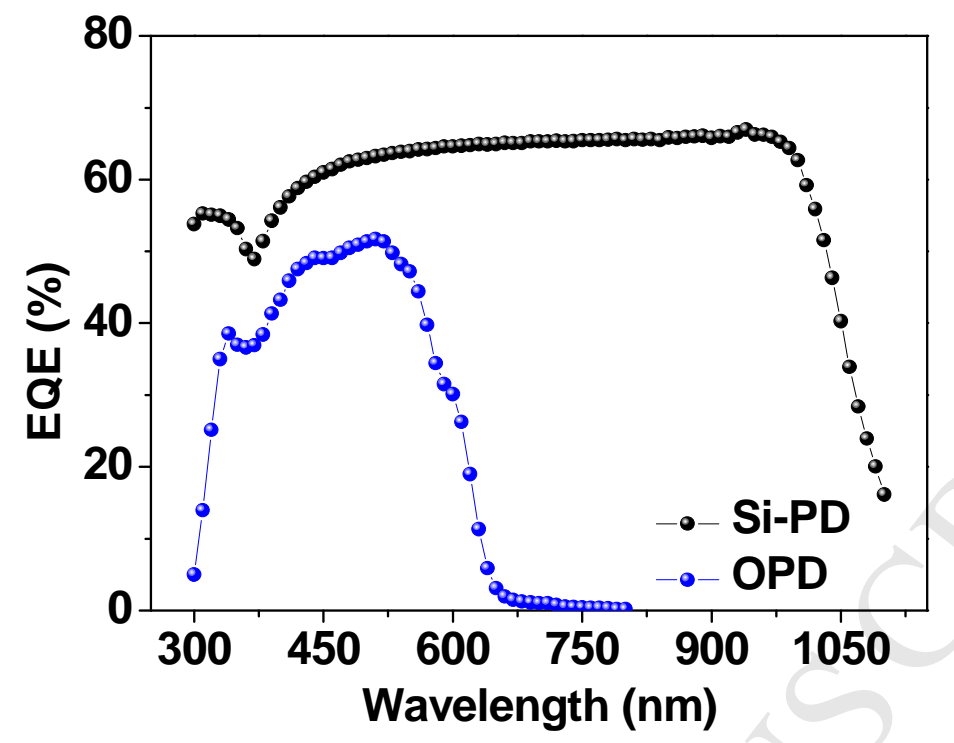

Fig. 4 Measured EQE results for Si-PD and OPD at zero bias voltage.

Figure 5 shows absorption and reflection simulation result in OPD obtained by GTMM. Note that, according to GTMM result, the absorption fraction of P3HT:PC 60 BM shows similar shape of EQE result in OPD. By comparing between EQE and GTMM results of P3HT:PC 60 BM, GTMM calculation would be useful to predict the OPD performance.

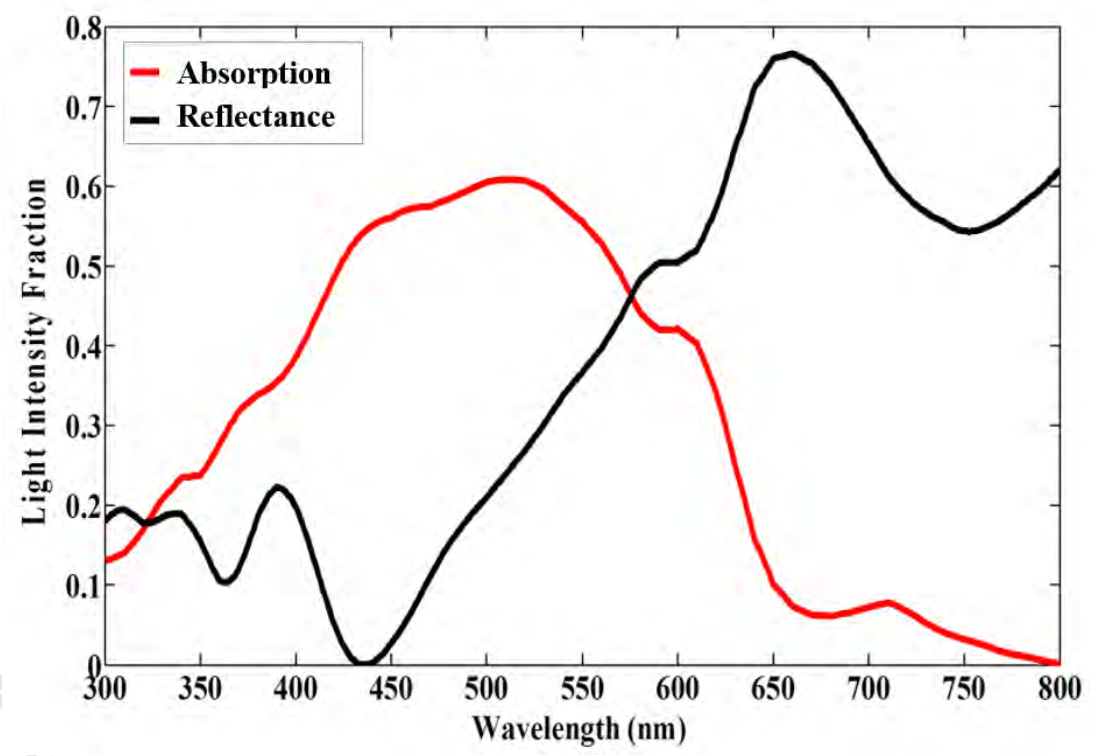

Fig. 5 Fraction of light absorption and reflection simulation result by generalized transfer matrix method (GTMM).

Figure 6 represents measured noise currents for both Si-PD and OPD at zero bias condition. Interestingly, both Si-PD and OPD shows that the major contribution of noise current comes from the 'flicker' noise, not the shot noise up to $10 \mathrm{~Hz}$. After $10 \mathrm{~Hz}$, the main contribution of the noise current is dominated by the combination of shot and thermal noise from the dark current. Furthermore, the dark current level at $0 \mathrm{~V}\left(\sim 10^{-11} \mathrm{~A} / \mathrm{cm}^{2}\right)$ and the noise current level after $10 \mathrm{~Hz}$ 
$\left(\sim 10^{-12} \mathrm{~A} / \mathrm{cm}^{2}\right)$ show a different level. Therefore, the noise spectra and noise current level should be addressed for the precision of the detectivity calculation.

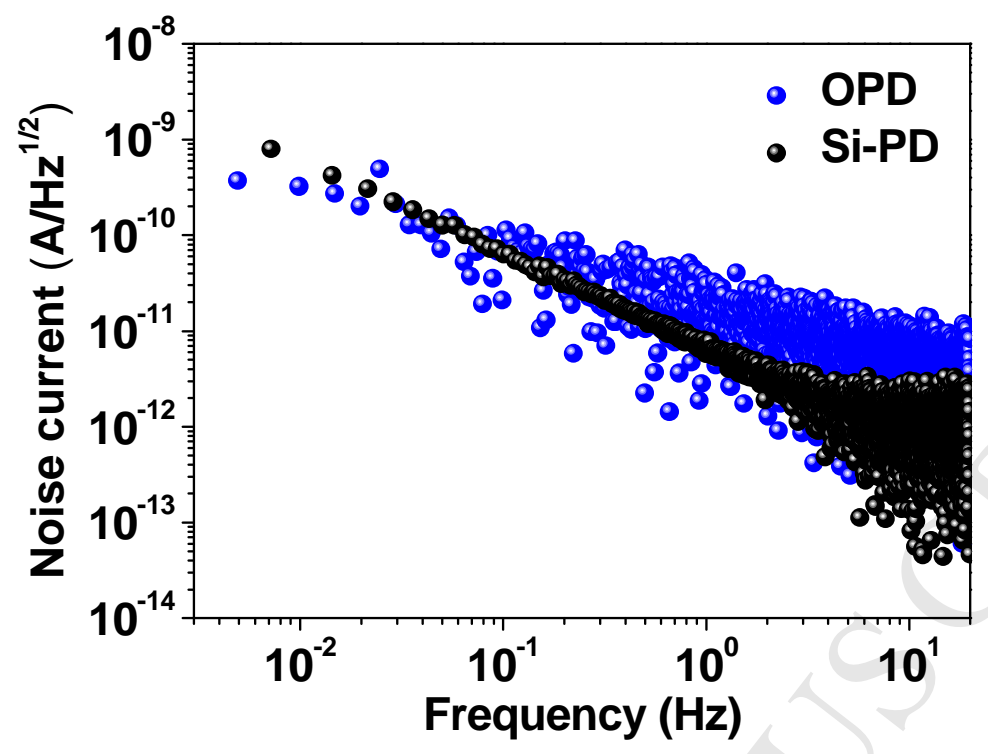

Fig. 6 Measured noise current results for Si-PD and OPD at zero bias voltage.

Figure 7 shows a comparison between dark current and noise current based detectivity spectra for both Si-PD and OPD, respectively. Similar to the literature [3-9], our dark current based spectrum demonstrates $\left(J_{d}=8.1 \times 10^{-11} \mathrm{~A} / \mathrm{cm}^{2}\right.$ and $D^{*}=4.22 \times 10^{13} \mathrm{cmHz}^{1 / 2} / \mathrm{W}$ at $520 \mathrm{~nm}$ for OPD and $J_{d}=$ $3.5 \times 10^{-11} \mathrm{~A} / \mathrm{cm}^{2}$ and $D^{*}=7.94 \times 10^{13} \mathrm{cmHz}^{1 / 2} / \mathrm{W}$ at $520 \mathrm{~nm}$ for Si-PD) a strong dependency on dark current at $0 \mathrm{~V}$ bias. However, the noise current based detectivity spectrum does not show such a high detectivity $\left(\sim 10^{13} \mathrm{cmHz}^{1 / 2} / \mathrm{W}\right.$ ), but it is in a reasonable range (up to $10^{11} \mathrm{cmHz}^{1 / 2} / \mathrm{W}$ for OPD and $10^{12} \mathrm{cmHz}^{1 / 2} / \mathrm{W}$ for Si-PD). For reliable detectivity, noise current is the main factor to analyze the detectivity with equation;

$$
D^{*}=(A \Delta f)^{1 / 2} R / i_{n}
$$

where $A$ is the effective area of the detector in $\mathrm{cm}^{2}, \Delta f$ is the electrical bandwidth in $\mathrm{Hz}$ and the $R$ the responsivity in $A / W$. 


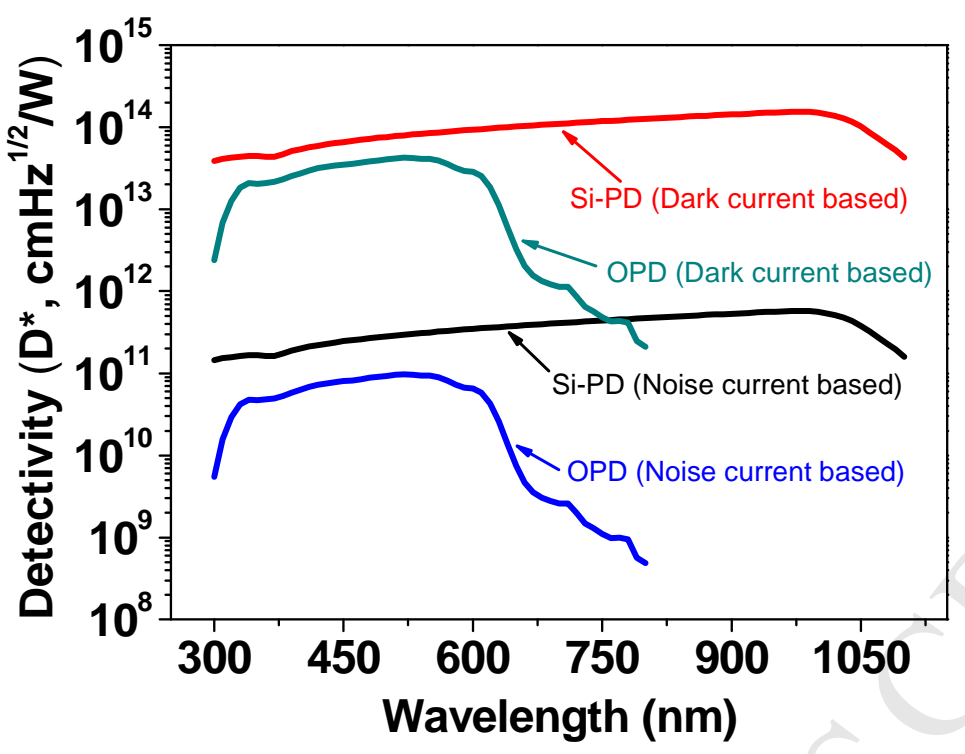

Fig. 7 Calculated detectivities of the Si-PD and OPD based on measured dark current $\left(J_{d}\right)$ and noise current $\left(i_{n}\right)$ at $0 \mathrm{~V}$ bias.

From the values of calculated detectivities, NEPs derived with the reciprocal of $D^{*}$ and can be expressed as

$$
\mathrm{NEP}=(A \Delta f)^{1 / 2} / D^{*}=i_{n} / R
$$

As mentioned above NEP equation (3), noise current in is still useful to calculate NEP values. Figure 8 shows both calculated NEPs. Calculated NEPs were $1.46 \times 10^{-11} \mathrm{~W} / \mathrm{Hz}^{1 / 2}$ at $520 \mathrm{~nm}$ for OPD and $5.51 \times 10^{-12} \mathrm{~W} / \mathrm{Hz}^{1 / 2}$ at $970 \mathrm{~nm}$ for Si-PD, respectively.

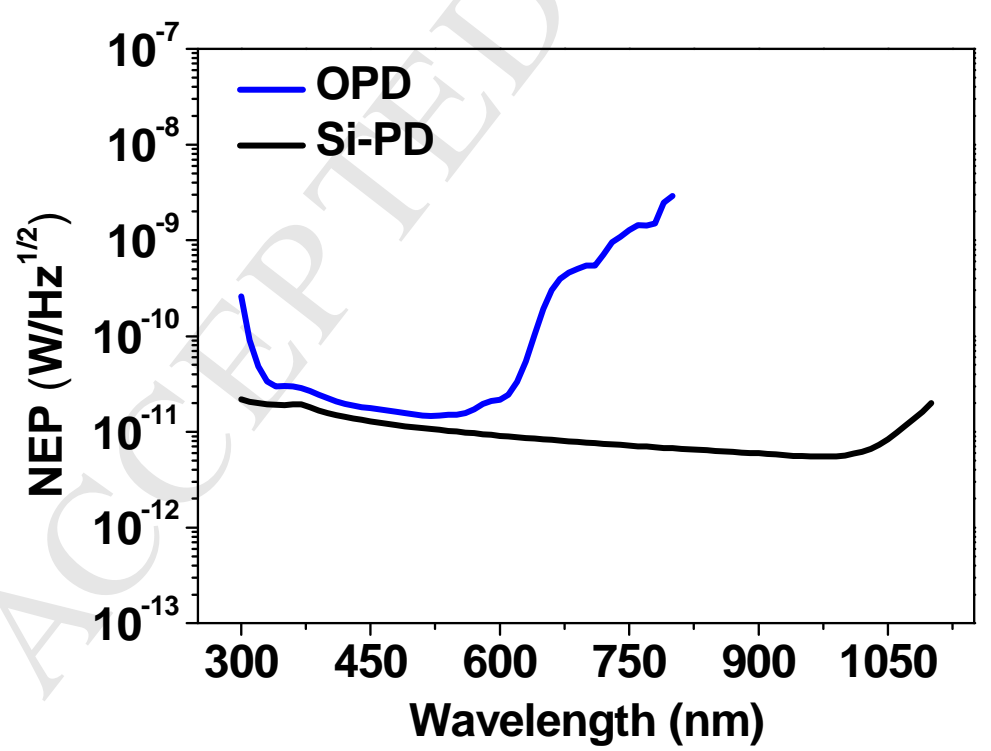

Fig. 8 Calculated NEPs of the Si-PD and OPD based on measured noise currents $\left(i_{n}\right)$ at $0 \mathrm{~V}$ bias.

\section{Conclusions}

In this perspective letter, we report a detectivity estimation problem based on dark current measurement. We found that a huge number of publications scaled their detectivities by the dark current measurements not by identifying the level of noise current. To address this issue, we have 
implemented this detectivity for both the organic photodiode and Si-photodiode. Finally, we found that there are huge differences (about 3 magnitudes of order) of detectivity calculations based on dark current measurement assumption and our noise measurement analysis.

\section{Acknowledgements}

This research was supported by the Pioneer Research Center Program through the National Research Foundation of Korea funded by the Ministry of Science, ICT \& Future Planning (2014M3C1A3053024).

\section{References}

[1] I. K. Kim, B. N. Pal, M. Ullah, P. L. Burn, S. C. Lo, P. Meredith, et al., "High-Performance, Solution-Processed Non-polymeric Organic Photodiodes," Advanced Optical Materials, vol. 3, pp. 50-56, 2015.

[2] G. Yu, K. Pakbaz, and A. Heeger, "Semiconducting polymer diodes: Large size, low cost photodetectors with excellent visible-ultraviolet sensitivity," Applied Physics Letters, vol. 64, pp. 3422-3424, 1994.

[3] X. Gong, M. Tong, Y. Xia, W. Cai, J. S. Moon, Y. Cao, et al., "High-detectivity polymer photodetectors with spectral response from $300 \mathrm{~nm}$ to $1450 \mathrm{~nm}$," Science, vol. 325, pp. 1665-1667, 2009.

[4] S.-J. Lim, D.-S. Leem, K.-B. Park, K.-S. Kim, S. Sul, K. Na, et al., "Organic-on-silicon complementary metal-oxide-semiconductor colour image sensors," Scientific reports, vol. 5, 2015.

[5] X. Hu, K. Wang, C. Liu, T. Meng, Y. Dong, S. Liu, et al., "High-detectivity inverted near-infrared polymer photodetectors using cross-linkable conjugated polyfluorene as an electron extraction layer," Journal of Material Chemistry C, vol. 2, pp. 9592-9598, 2014.

[6] Y. J. Kim, C. E. Park, and D. S. Chung, "Interface engineering of a highly sensitive solution processed organic photodiode," Physical Chemistry Chemical Physics, vol. 16, pp. 18472-18477, 2014.

[7] Z. Liu, K. Parvez, R. Li, R. Dong, X. Feng, and K. Müllen, "Transparent Conductive Electrodes from Graphene/PEDOT: PSS Hybrid Inks for Ultrathin Organic Photodetectors," Advanced Materials, 2014.

[8] D.-H. Kim, K.-S. Kim, H.-S. Shim, C.-K. Moon, Y. W. Jin, and J.-J. Kim, "A high performance semitransparent organic photodetector with green color selectivity," Applied Physics Letters, vol. 105, p. 213301, 2014.

[9] S. Shafian, Y. Jang, and K. Kim, "Solution processed organic photodetector utilizing an interdiffused polymer/fullerene bilayer," Optics Express, vol. 23, pp. A936-A946, 2015.

[10] Burkhard. G. F, Hoke. E.T, McGehee. M.D, "Accounting for interference, scattering, and electrode absorption to make accurate internal quantum efficiency measurements in organic and other thin solar cells". Advanced Materials, 22, 3293-3297. 2010

[11] Zhao. X, Li. Z, Zhu. T, Mi. B, Gao. Z, Huang. W. "Structure optimization of organic planar heterojunction solar cells". Journal of Physics D. 46. 2013

[12] I. K. Kim, X. Li, M. Ullah, P. E. Shaw, R. Wawrzinek, E. B. Namdas, et al., "High-Performance, Fullerene-Free Organic Photodiodes Based on a Solution-Processable Indigo," Advanced Materials, vol. 27, pp. 6390-6395, 2015. 
Dear Editor,

We are submitting a manuscript below, which is an outcome of our original research work. We have carefully prepared the manuscript and would like to request your kind consideration for publication in "Organic Electronics". Below is the highlight of the manuscript.

In this perspective letter, we report a detectivity estimation problem based on dark current measurement. We found that a huge number of publications scaled their detectivities by the dark current measurements not by identifying the level of noise current. To address this issue, we have implemented this detectivity for both the organic photodiode and Si-photodiode. Finally, we found that there are huge differences (about 3 magnitudes of order) of detectivity calculations based on dark current measurement assumption and our noise measurement analysis. For the above reasons, we firmly believe that it provides a new foundation for organic photodiode research.

Best regards,

Il Ku Kim, Ph. D.

Department of Nanotechnology and Advanced Materials Engineering

Sejong University

143-147 Seoul, Korea 


\section{Accepted Manuscript}

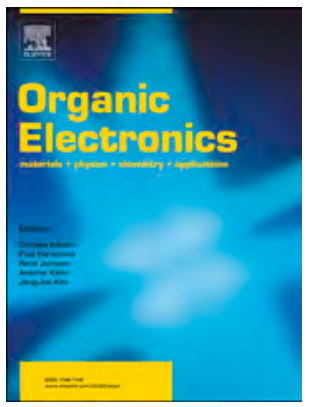

PII:

S1566-1199(18)30090-9

DOI:

10.1016/j.orgel.2018.02.036

Reference:

ORGELE 4550

To appear in: Organic Electronics

Received Date: 1 August 2017

Revised Date: 29 January 2018

Accepted Date: 23 February 2018

Please cite this article as: I.K. Kim, J.H. Jo, B.J. Lee, Y.J. Choi, Detectivity analysis for organic photodetectors, Organic Electronics (2018), doi: 10.1016/j.orgel.2018.02.036.

This is a PDF file of an unedited manuscript that has been accepted for publication. As a service to our customers we are providing this early version of the manuscript. The manuscript will undergo copyediting, typesetting, and review of the resulting proof before it is published in its final form. Please note that during the production process errors may be discovered which could affect the content, and all legal disclaimers that apply to the journal pertain. 